\title{
Fauna and Flora of Forests Over Limestone in Calicoan Island, Guiuan Marine Reserve Protected Landscape and Seascape (GMRPLS), Eastern Samar, Philippines
}

\author{
Desamarie Antonette P. Fernandez \\ (corresponding author) \\ Animal Biology Division, Institute of Biological Sciences, College \\ of Arts and Sciences; CONserve KAIGANGAN Program, \\ University of the Philippines Los Baños, College, Laguna, \\ Philippines \\ dpfernandez1@up.edu.ph
}

\section{Ren Divien R. Obeña}

CONserve KAIGANGAN Program, Institute of Biological Sciences, University of the Philippines Los Baños, College, Laguna, Philippines

\section{Elaine Loreen C. Villanueva}

CONserve KAIGANGAN Program, Institute of Biological Sciences, University of the Philippines Los Baños, College, Laguna, Philippines

\author{
Marjorie D. delos Angeles \\ Plant Biology Division, Institute of Biological Sciences, College of \\ Arts and Sciences; CONserve KAIGANGAN Program, University \\ of the Philippines Los Baños, College, Laguna, Philippines \\ Center of Integrative Conservation, Xishuangbanna Tropical \\ Botanical Garden, Chinese Academy of Sciences, Menglun, \\ Mengla, Yunnan, China
}

\author{
Paul John S. Tolentino \\ CONserve KAIGANGAN Program, Institute of Biological \\ Sciences, University of the Philippines Los Baños, College, \\ Laguna, Philippines
}

Inocencio E. Buot Jr.

Plant Biology Division, Institute of Biological Sciences, College of Arts and Sciences; CONserve KAIGANGAN Program, University of the Philippines Los Baños, College, Laguna, Philippines

Publication Information:

Received 21 July 2020, Accepted 18 October 2020, Available online 22 December 2020

DOI: 10.21463/jmic.2020.09.2.07

\section{Abstract}

This study presents the first checklist of the lesser known terrestrial biodiversity in forests over limestone karst of Calicoan Island in Guiuan Marine Reserve Protected Landscape and Seascape, Guiuan, Eastern Samar, Philippines. Plants and terrestrial vertebrate species in nine $20 \times 20 \mathrm{~m}$ plots and 18 line transects were recorded and identified. A total of 60 bird, eight reptile, one amphibian, and six mammal species were recorded. Moreover, a total of 41 floral species were documented belonging to 17 plant families and 24 genera. Of the plant species recorded, 5 were shrubs and 35 were trees. This study reported a new locality record of the Philippine endemic tree species Hancea wenzeliana, and new island records for fauna such as Varanus samarensis and Cyrtodactylus sumuroi, among many others. To date, the 
present study is the only assessment of herpetofauna and mammals in Guiuan and represent new island records for most of these taxa in Calicoan Island. Among plants, Shorea negrosensis, Aquilaria cuminigiana and Wallaceodendron celebicum were identified to have a vulnerable conservation status based on their IUCN Red List and DENR-DAO 2017-11. These native and endemic plants can be used for reforestation programs in the area, and conserving biodiversity in general will be crucial to potential ecotourism programs.

\section{Keywords}

Guiuan, kaigangan, forest over limestone, biodiversity, ecotourism, resiliency

\section{Introduction}

Forests over limestone are major foci for speciation and important biodiversity arks (Day \& Urich, 2000) which contain unique biodiversity because of their unique topology. Biological diversity is a significant driver towards nature conservation (Myers et al., 2000) and is responsible for various ecosystem services which contribute to human wellbeing and survival, among others. Despite its high species endemicity and environmental heterogeneity, forests over limestone are more vulnerable since they recover slower due to relatively dry habitat and shallow soil, which can be irreversible once damaged (Tuyet, 2001).

Guiuan Marine Reserve Protected Landscape and Seascape (GMRPLS) in Eastern Samar, Philippines was designated as a protected area by virtue of Presidential Proclamation No. 469 in 1994. It is notable for its rich marine resources which are utilized by local residents, the academe and surfers. It may be rich in terrestrial and marine resources, but most research conducted in the area were focused on marine plants and algae (Peja et al., 2018; Corales-Ultra et al., 2019), mangrove communities along the coastline of Eastern Samar (Mendoza and Alura, 2001; Alura and Alura, 2016), and assessment of household resiliency (Hilvano et al., 2016) after Typhoon Haiyan (local name Yolanda). This typhoon was a fast-moving and extremely intense tropical cyclone which devastated the island in the year 2013 (Lin et al., 2014; Takagi and Esteban, 2016).

Most of the terrestrial forest areas of this protected area remain largely unexplored and biological knowledge is deficient. There are currently no published studies with emphasis on the terrestrial biodiversity of the forests over limestone of GMRPLS. Additional efforts for biodiversity documentation and forest ecosystem monitoring of the area will be a way to address the gaps. Thus, there is a need for collections and updates of faunal and floristic inventories. These are important especially in areas such as forest over limestone which require proper conservation, monitoring, and management. Knowledge in terrestrial biodiversity can lead to proper management practices to protect these forests from threats, abusive land use patterns (Sodhi et al., 2010), alterations, and climate change which would be of great help, specifically for GMRPLS, given that its geographic position that faces the Pacific Ocean making the entire protected area prone to typhoons, storm surges and tsunamis.

This study aimed to evaluate the faunal and floral composition of forests over limestone in GMRPLS with notes on conservation and distribution, and to discuss the value of terrestrial biodiversity to resilience and ecotourism. The study will provide biological reference in the form of a checklist for future ecological research, conservation, and monitoring efforts. 


\title{
Materials and Methods
}

\author{
Study area
}

Guiuan Marine Reserve Protected Landscape and Seascape (GMRPLS) can be found in the southeastern tip of Samar Island facing the Pacific Ocean (Fig. 1). It is located in the municipality of Guiuan, in the province of Eastern Samar, Philippines, approximately $10^{\circ} 58^{\prime} 57.1^{\prime \prime} \mathrm{N}$ latitude and $125^{\circ} 48^{\prime} 10.9^{\prime \prime} \mathrm{E}$ longitude. It has a total area of 60,448 hectares covering the coastal areas of Guiuan and nearby islands of Calicoan, Manicani, Candulo, Suluan, Tubabao, and Homonhon and surrounding reefs.

The selection of sampling plots was based on the biophysical characteristics of the area including plant diversity heterogeneity, topographic attribute (elevation), extent of forest over limestone area, and presence of anthropogenic disturbances. An assessment of the faunal and floral diversity of GMRPLS was conducted on October 8-11, 2019, as part of Project 1: Assessment of Biodiversity in Forest Over Limestone Ecosystem. This is a component of the government-funded research program, Assessment and Conservation of Forest Over Limestone Ecosystem Biodiversity in Selected Municipalities of Samar, Philippines (CONserve-KAIGANGAN). Since Calicoan Island has the largest continuous forest over limestone area in GMRPLS, nine 20x20 m vegetation plots were deployed across its four barangays (abbreviated as brgy., the Filipino term for a district): Baras, Ngolos, Pagnamitan and Sulangan.

\section{Faunal diversity assessment}

Various methods adopted from the Manual on Biodiversity Assessment and Monitoring System for Terrestrial Ecosystems (Cruz et al., 2017) were used to assess terrestrial vertebrate diversity within the established plots and transects. Herpetofauna, which includes amphibians and reptiles, were hand-captured within the sampling plots or collected using forceps, snake hook/tongs and placed in separate labeled resealable plastic bags for easier identification. All individuals were immediately released after species identification.

A simple line transect count was done for the survey of birds. Binoculars, field guides such as those by Kennedy et al. (2000) and Arlott (2018), as well as online references such as Handbook of the Birds of the World Alive (del Hoyo et al., 2020), were used to aid in species identification. Birds were photographed using DSLR camera with telephoto lens and a digital audio-recorder was used for recording bird calls.

To capture birds and volant mammals, 12-meter mist nets were set along possible flyways and feeding trees in or around the sampling plots. These are nylon nets with $35 \mathrm{~mm}$ sized mesh, set in 3-4 rungs with loose pockets to allow capture. Mist nets were checked periodically for possible netted individuals every hour from 1830 to $2200 \mathrm{~h}$. Nets are opened for 24 hours for at least three days and two nights. Each captured animal was carefully removed from the net and placed individually in cloth bags to minimize stress and injury. All individuals were immediately released after species identification. 


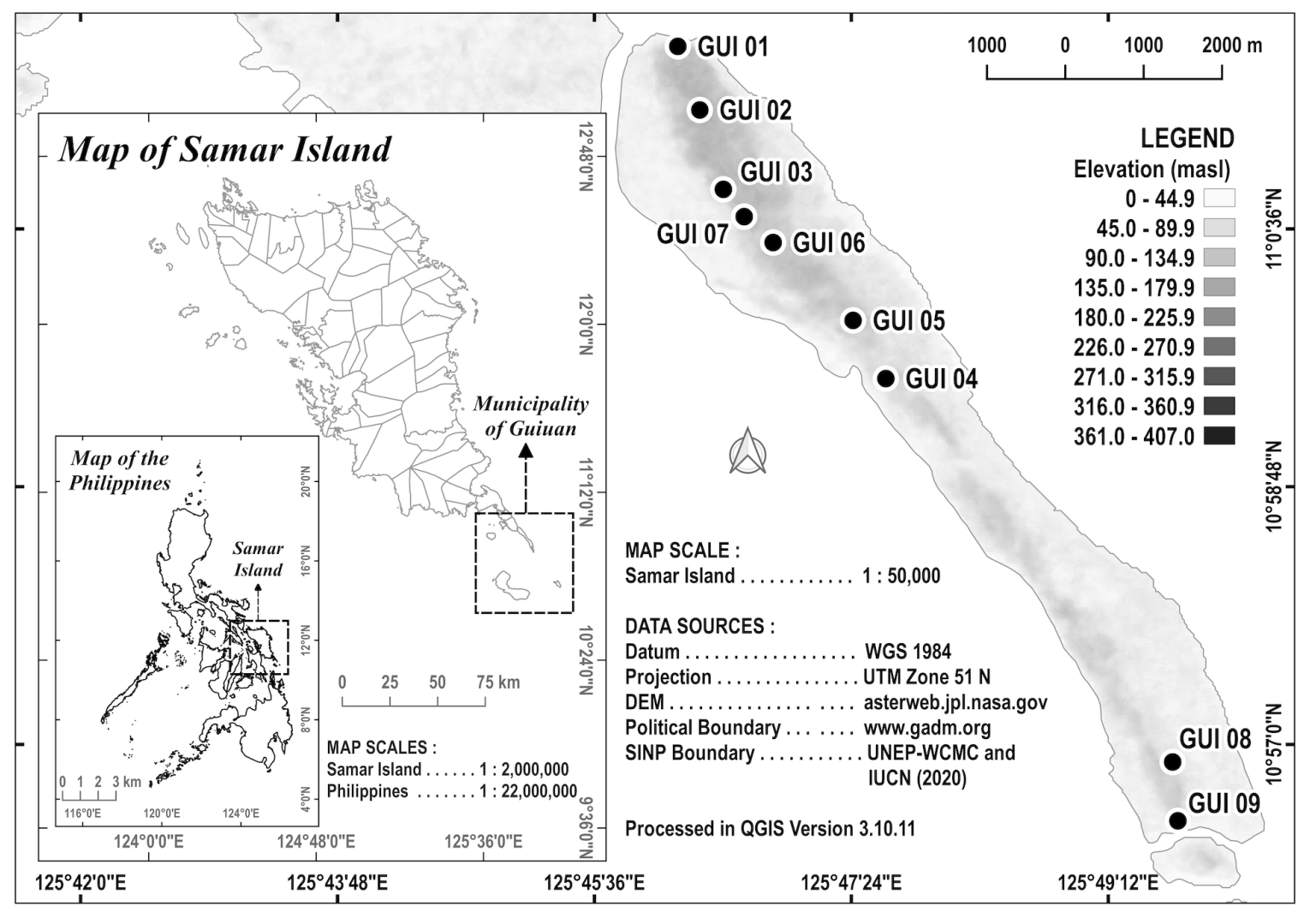

Fig 1. Map of Guiuan Marine Reserve Protected Landscape and Seascape (GMRPLS), Philippines. Established plots are marked in black circles.

Steel mesh cage traps were used to capture small non-volant mammals. To prepare bait, thinly sliced coconut meat was fried until brown and coated generously with peanut butter. Live earthworms also served as bait when available. Rat traps were set within sampling plots and along transects. For trapping on the ground surface, the traps were positioned 5-10 m apart under root tangles, in front of burrow entrances, along runways, and beside or on top of fallen logs. For aboveground trapping, the traps were tied onto horizontal branches of trees. Captures were retrieved early in the morning (c. a. $0700 \mathrm{~h}$ ) and baits were replaced in the late afternoon (c.a. 1700 h). All individuals were immediately released after species identification. Camera traps were also set up to photograph large or cryptic species.

\section{Floral diversity assessment}

Two types of methods were used to determine the plant species composition of each sampling site. The quadrat or plot technique was used to assess the trees $(\geq 1 \mathrm{~m})$, while line intercept technique was used for understory plant species. Trees and shrubs inside each plot were recorded. Coordinates and elevation of sampling plots were taken using the Garmin Montana 680 Global Positioning System (GPS). A total of 9 plots and 18 line transects were established in Guiuan to assess trees and the understory species, respectively. Two line transects, $5 \mathrm{~m}$ in length and subdivided with 1 $\mathrm{m}$ intervals, were deployed inside each sampling plot. The local name, height, and cover of all plant species transverse in the line were recorded per $1 \mathrm{~m}$ interval markings. 


\section{Herbarium specimen}

Voucher specimens were collected for proper identification and documentation. These were then processed, and herbarium specimens were deposited in the Plant Biology Division Herbarium (PBDH), Institute of Biological Sciences, University of the Philippines Los Baños. Specimens were identified utilizing original descriptions, taxonomic revisions, and botanical keys. Our specimens were also compared with the collections of PBDH and from available online resources such as “Co's Digital Flora of the Philippines” (Pelser et al., 2011). Because existing databases and updated information regarding the flora of Samar are wanting, experts from the Philippine National Museum were consulted in order to identify the unknown plant specimens. Voucher information for the specimens, geographic coordinates of collection sites, life form, and endemicity were also noted. Nomenclature follows that of Pelser et al. (2011).

\section{Secondary data collection}

Published literature, monographs, and other checklists regarding biodiversity in Guiuan were also reviewed in relation to species occurrence and endemicity. The endemic status of each species was verified by consulting available checklists, related publications, and online resources.

The conservation status of each animal species was determined from the International Union for the Conservation of Nature (IUCN) Red List and the Department of Environment and Natural Resources Administrative Order (DAO) 2019-09 or the Updated National List of Threatened Philippine Fauna and Their Categories (DENR, 2019). Meanwhile, the IUCN Red List conservation status of each plant species was determined from "Co's Digital Flora of the Philippines" (Pelser et al., 2011) and the DAO 2017-11 or the Updated National List of Threatened Philippine Plants and Their Categories (DENR, 2017).

\section{Results}

\section{Faunal composition of tropical forest over limestone}

A total of one amphibian, eight reptile, 60 bird, and six mammal species were recorded during the fieldwork (Table 1). The only amphibian species found, Platymantis guentheri, is endemic to the Mindanao Pleistocene Aggregate Island Complex (PAIC). Four of the reptile species, Draco bimaculatus, Sphenomorphus jagori, Cyrtodactylus sumuroi, and Varanus samarensis, are Philippine endemics, the last two of which are Mindanao PAIC endemics. Eleven bird species are known to be migratory species, and two species, Phylloscopus olivaceus and Hypsipetes philippinus saturatior, are Philippine endemics, the latter of which is a Mindanao PAIC endemic subspecies. Two of the volant mammal species, Ptenochirus jagori and Ptenochirus minor, are Philippine endemics, the latter of which is a Mindanao PAIC endemic. The two non-volant mammal species, Rattus everetti and Bullimus bagobus, are Philippine endemics, the latter of which is a Mindanao PAIC endemic.

Among these bird species, only 6 were documented during the field assessment. The majority of avian records in this checklist were from secondary sources such as Potter (1953) and Canillas (2018a, 2018b, 2019). Although Potter 
documented the presence of the critically endangered Cacatua haematuropygia in 1953, they may now be extinct in the region (BirdLife International, 2017), although further study is required to verify this information.

Table 1. List of terrestrial vertebrate species recorded from the forests over limestone of Guiuan Marine Reserve Protected Landscape and Seascape in Guiuan, Eastern Samar, Philippines.

\begin{tabular}{|c|c|c|c|c|c|c|c|}
\hline \multirow[b]{2}{*}{ Order } & \multirow[b]{2}{*}{ Family } & \multirow[b]{2}{*}{ Species } & \multirow[b]{2}{*}{ Common Name } & \multirow[b]{2}{*}{ Distribution } & \multicolumn{2}{|c|}{ Conservation Status* } & \multirow[b]{2}{*}{ Reference } \\
\hline & & & & & $\begin{array}{l}\text { IUCN Red } \\
\text { List }\end{array}$ & $\begin{array}{c}\text { DAO } \\
2019-09\end{array}$ & \\
\hline \multicolumn{8}{|c|}{ Amphibians } \\
\hline Anura & Ceratobatrachidae & Platymantis guentheri & $\begin{array}{l}\text { Gunther's wrinkled ground } \\
\text { frog }\end{array}$ & Endemic & LC & None & Fieldwork \\
\hline \multicolumn{8}{|c|}{ Reptiles } \\
\hline \multirow[t]{8}{*}{ Squamata } & \multirow[t]{2}{*}{ Agamidae } & Bronchocela cristatella & Green crested lizard & Resident & None & OTS & Fieldwork \\
\hline & & Draco bimaculatus & Two-spotted flying lizard & Endemic & LC & None & Fieldwork \\
\hline & \multirow[t]{2}{*}{ Scincidae } & $\begin{array}{l}\text { Lamprolepis smaragdina } \\
\text { philippinica }\end{array}$ & Emerald tree skink & Resident & None & None & Fieldwork \\
\hline & & Sphenomorphus jagori & Jagor's sphenomorphus & Endemic & LC & None & Fieldwork \\
\hline & Gekkonidae & Cyrtodactylus sumuroi & Samar bent-toed gecko & Endemic & None & None & Fieldwork \\
\hline & Pythonidae & Malayopython reticulatus & Reticulated python & Resident & LC & OTS & Fieldwork \\
\hline & Viperidae & Tropidolaemus subannulatus & Keeled Green Pit Viper & Resident & LC & OTS & Fieldwork \\
\hline & Varanidae & Varanus samarensis & Samar water monitor & Endemic & None & OTS & Fieldwork \\
\hline \multicolumn{8}{|c|}{ Birds } \\
\hline \multirow[t]{4}{*}{ Pelecaniformes } & \multirow[t]{4}{*}{ Ardeidae } & Ardea intermedia & Intermediate egret & Migratory & LC & None & $\begin{array}{l}\text { Canillas } \\
2018 a\end{array}$ \\
\hline & & Butorides striata & Green-backed heron & Migratory & LC & None & Potter 1953 \\
\hline & & Egretta sacra & Pacific reef heron & Resident & LC & None & Potter 1953 \\
\hline & & Nycticorax caledonicus & Rufous night heron & Resident & LC & None & $\begin{array}{l}\text { Canillas } \\
2019\end{array}$ \\
\hline \multirow[t]{4}{*}{ Accipitriformes } & \multirow[t]{3}{*}{ Accipitridae } & Haliastur indus & Braminy kite & Resident & LC & None & Potter 1953 \\
\hline & & Accipiter trivirgatus & Crested goshawk & Resident & LC & None & Potter 1953 \\
\hline & & Spilornis holospilus & Philippine serpent eagle & Endemic & LC & None & Potter 1953 \\
\hline & Falconidae & Microhierax erythrogenys & Philippine falconet & Endemic & LC & None & Potter 1953 \\
\hline \multirow[t]{9}{*}{ Charadriiformes } & \multirow[t]{2}{*}{ Charadriidae } & Charadrius mongolus & Lesser sand plover & Migratory & LC & None & $\begin{array}{l}\text { Canillas } \\
2018 a\end{array}$ \\
\hline & & Pluvialis fulva & Pacific golden plover & Migratory & LC & None & Potter 1953 \\
\hline & \multirow[t]{7}{*}{ Scolopacidae } & Calidris minuta & Little stint & Resident & LC & None & Potter 1953 \\
\hline & & Limosa lapponica & Bar-tailed godwit & Migratory & NT & None & $\begin{array}{l}\text { Canillas } \\
2018 b\end{array}$ \\
\hline & & Numenius madagascariensis & Far Eastern curlew & Migratory & EN & None & $\begin{array}{l}\text { Canillas } \\
2018 b\end{array}$ \\
\hline & & Numenius phaeopus & Eurasian whimbrel & Migratory & LC & None & Potter 1953 \\
\hline & & Tringa brevipes & Grey-tailed tattler & Migratory & NT & None & Potter 1953 \\
\hline & & Tringa totanus & Common redshank & Migratory & LC & None & $\begin{array}{l}\text { Canillas } \\
2019\end{array}$ \\
\hline & & Phalaropus lobatus & Red-necked phalarope & Migratory & LC & None & Potter 1953 \\
\hline
\end{tabular}




\begin{tabular}{|c|c|c|c|c|c|c|c|}
\hline \multirow[b]{2}{*}{ Order } & \multirow[b]{2}{*}{ Family } & \multirow[b]{2}{*}{ Species } & \multirow[b]{2}{*}{ Common Name } & \multirow[b]{2}{*}{ Distribution } & \multicolumn{2}{|c|}{ Conservation Status* } & \multirow[b]{2}{*}{ Reference } \\
\hline & & & & & $\begin{array}{l}\text { IUCN Red } \\
\text { List }\end{array}$ & $\begin{array}{c}\text { DAO } \\
2019-09\end{array}$ & \\
\hline \multirow[t]{2}{*}{ Columbiformes } & \multirow[t]{2}{*}{ Columbidae } & $\begin{array}{l}\text { Phapitreron brevirostris } \\
\text { brevirostris }\end{array}$ & Short-billed brown-dove & Endemic & LC & None & Potter 1953 \\
\hline & & Ducula aenea & Green imperial-pigeon & Resident & LC & None & Potter 1953 \\
\hline \multirow[t]{4}{*}{ Cuculiformes } & \multirow[t]{4}{*}{ Cuculidae } & Cuculus saturatus & Oriental cuckoo & Resident & LC & None & Potter 1953 \\
\hline & & Cuculus canorus & Common cuckoo & Migratory & LC & None & Potter 1953 \\
\hline & & Eudynamys scolopaceus & Western koel & Resident & LC & None & Potter 1953 \\
\hline & & Centropus viridis viridis & Philippine Coucal & Endemic & LC & None & Potter 1953 \\
\hline \multirow[t]{6}{*}{ Coraciiformes } & \multirow[t]{4}{*}{ Alcedinidae } & Alcedo atthis bengalensis & Common kingfisher & Resident & LC & None & Potter 1953 \\
\hline & & Halcyon coromanda & Ruddy kingfisher & Migratory & LC & None & Fieldwork \\
\hline & & $\begin{array}{l}\text { Todiramphus winchelli } \\
\text { nigrorum }\end{array}$ & Rufous-lored kingfisher & Endemic & VU & VU & Potter 1953 \\
\hline & & Todiramphus chloris & Collared kingfisher & Resident & LC & None & Potter 1953 \\
\hline & Coraciidae & Eurystomus orientalis & Oriental dollarbird & Resident & LC & None & Potter 1953 \\
\hline & Meropidae & Merops philippinus & Blue-tailed bee-eater & Resident & LC & None & Potter 1953 \\
\hline \multirow[t]{2}{*}{ Bucerotiformes } & \multirow[t]{2}{*}{ Bucerotidae } & $\begin{array}{l}\text { Buceros mindanensis } \\
\text { semigaleatus }\end{array}$ & $\begin{array}{l}\text { Southern rufous } \\
\text { hornbill }\end{array}$ & Endemic & VU & EN & Potter 1953 \\
\hline & & Penelopides affinis & Mindanao hornbill & Endemic & LC & EN & Potter 1953 \\
\hline \multirow[t]{3}{*}{ Piciformes } & \multirow[t]{3}{*}{ Picidae } & $\begin{array}{l}\text { Picoides maculatus } \\
\text { fulvifasciatus }\end{array}$ & $\begin{array}{l}\text { Philippine pygmy } \\
\text { woodpecker }\end{array}$ & Endemic & LC & None & Potter 1953 \\
\hline & & $\begin{array}{l}\text { Chrysocolaptes lucidus } \\
\text { rufopunctatus }\end{array}$ & Buff-spotted flameback & Endemic & LC & None & Potter 1953 \\
\hline & & Dryocopus javensis pectoralis & $\begin{array}{l}\text { White-bellied } \\
\text { woodpecker }\end{array}$ & Resident & LC & None & Potter 1953 \\
\hline \multirow[t]{3}{*}{ Psittaciformes } & Cacatuidae & Cacatua haematuropygia & Philippine cockatoo & Endemic & $\mathrm{CR}$ & $\mathrm{CR}$ & Potter 1953 \\
\hline & \multirow[t]{2}{*}{ Psittacidae } & Loriculus philippensis & Colasisi & Endemic & LC & $\mathrm{CR}$ & Potter 1953 \\
\hline & & $\begin{array}{l}\text { Prioniturus discurus } \\
\text { whiteheadi }\end{array}$ & $\begin{array}{l}\text { Blue-crowned racket- } \\
\text { tail }\end{array}$ & Endemic & LC & OTS & Potter 1953 \\
\hline \multirow[t]{11}{*}{ Passeriformes } & Artamidae & $\begin{array}{l}\text { Artamus leucorynchus } \\
\text { leucorhynchus }\end{array}$ & $\begin{array}{l}\text { White-breasted } \\
\text { woodswallow }\end{array}$ & Resident & LC & None & Potter 1953 \\
\hline & Campephagidae & Coracina striata boholensis & $\begin{array}{l}\text { Bar-bellied } \\
\text { cuckooshrike }\end{array}$ & Resident & LC & None & Potter 1953 \\
\hline & Cisticolidae & Orthotomus frontalis frontalis & $\begin{array}{l}\text { Rufous-fronted } \\
\text { tailorbird }\end{array}$ & Endemic & LC & None & Potter 1953 \\
\hline & Corvidae & Corvus macrorhynchos & Large-billed crow & Resident & LC & OTS & Potter 1953 \\
\hline & \multirow[t]{2}{*}{ Dicaeidae } & Dicaeum papuense papuense & Philippine Flowerpecker & Resident & LC & None & Potter 1953 \\
\hline & & $\begin{array}{l}\text { Dicaeum trigonostigma } \\
\text { cinereigulare }\end{array}$ & $\begin{array}{l}\text { Grey-throated } \\
\text { flowerpecker }\end{array}$ & Resident & LC & None & Potter 1953 \\
\hline & Dicruridae & Dicrurus striatus & Short-tailed drongo & Endemic & LC & None & Potter 1953 \\
\hline & Laniidae & Lanius cristatus lucionensis & Brown shrike & Resident & LC & None & $\begin{array}{l}\text { Fieldwork, Potter } \\
1953\end{array}$ \\
\hline & Hirundinidae & Hirundo javanica & House swallow & Resident & LC & None & Potter 1953 \\
\hline & \multirow[t]{2}{*}{ Motacillidae } & Anthus gustavi & Pechora pipit & Migratory & LC & None & Potter 1953 \\
\hline & & Motacilla tschutschensis & Eastern yellow wagtail & Resident & LC & None & Potter 1953 \\
\hline
\end{tabular}




\begin{tabular}{|c|c|c|c|c|c|c|c|}
\hline \multirow[b]{2}{*}{ Order } & \multirow[b]{2}{*}{ Family } & \multirow[b]{2}{*}{ Species } & \multirow[b]{2}{*}{ Common Name } & \multirow[b]{2}{*}{ Distribution } & \multicolumn{2}{|c|}{ Conservation Status* } & \multirow[b]{2}{*}{ Reference } \\
\hline & & & & & $\begin{array}{c}\text { IUCN Red } \\
\text { List }\end{array}$ & $\begin{array}{l}\text { DAO } \\
2019-09\end{array}$ & \\
\hline & \multirow[t]{3}{*}{ Muscicapidae } & $\begin{array}{l}\text { Cyornis rufigastra } \\
\text { philippinensis }\end{array}$ & $\begin{array}{l}\text { Mangrove blue- } \\
\text { flycatcher }\end{array}$ & Resident & LC & None & Fieldwork \\
\hline & & $\begin{array}{l}\text { Monticola solitarius } \\
\text { philippensis }\end{array}$ & $\begin{array}{l}\text { Eastern blue rock- } \\
\text { thrush }\end{array}$ & Resident & LC & None & $\begin{array}{l}\text { Fieldwork, Potter } \\
1953\end{array}$ \\
\hline & & Muscicapa griseisticta & $\begin{array}{l}\text { Gray-streaked } \\
\text { flycatcher }\end{array}$ & Resident & LC & None & Potter 1953 \\
\hline & Nectariniidae & Leptocoma sperata trochilus & Purple-throated sunbird & Endemic & LC & None & Potter 1953 \\
\hline & Oriolidae & Oriolus chinensis & Black-naped oriole & Resident & LC & None & Potter 1953 \\
\hline & \multirow[t]{2}{*}{ Phylloscopidae } & Phylloscopus borealis & Arctic warbler & Resident & LC & None & Potter 1953 \\
\hline & & Phylloscopus olivaceus & Philippine leaf-warbler & Endemic & LC & None & Fieldwork \\
\hline & Pycnonotidae & $\begin{array}{l}\text { Hypsipetes philippinus } \\
\text { saturatior }\end{array}$ & Philippine bulbul & Endemic & LC & None & $\begin{array}{l}\text { Fieldwork, Potter } \\
1953\end{array}$ \\
\hline & \multirow[t]{3}{*}{ Sturnidae } & Aplonis panayensis & Asian glossy starling & Resident & LC & None & Potter 1953 \\
\hline & & Sarcops calvus & Coleto & Endemic & LC & None & Potter 1953 \\
\hline & & Rhabdornis mystacalis minor & $\begin{array}{l}\text { Stripe-headed } \\
\text { rhabdornis }\end{array}$ & Endemic & LC & None & Potter 1953 \\
\hline & Zosteropidae & $\begin{array}{l}\text { Zosterops palpebrosus } \\
\text { basilanica }\end{array}$ & Oriental White-eye & Resident & LC & None & Potter 1953 \\
\hline \multicolumn{8}{|c|}{ Mammals } \\
\hline \multirow[t]{3}{*}{ Chiroptera } & \multirow[t]{3}{*}{ Pteropodidae } & Ptenochirus jagori & Greater musky fruit bat & Endemic & LC & None & Fieldwork \\
\hline & & Ptenochirus minor & Lesser musky fruit bat & Endemic & LC & None & Fieldwork \\
\hline & & Rousettus amplexicaudatus & Geoffrey's rousette & Resident & LC & None & Fieldwork \\
\hline Primates & Cercopithecidae & Macaca fascicularis & Long-tailed macaque & Resident & LC & None & Fieldwork \\
\hline \multirow[t]{2}{*}{ Rodentia } & \multirow[t]{2}{*}{ Muridae } & Rattus everetti & Philippine forest rat & Endemic & LC & None & Fieldwork \\
\hline & & Bullimus bagobus & Bagobo rat & Endemic & LC & None & $\begin{array}{l}\text { Fieldwork, } \\
\text { Johnson } 1946\end{array}$ \\
\hline
\end{tabular}

${ }^{*}$ CR- Critically Endangered, EN- Endangered, VU- Vulnerable, NT- Near Threatened, LC- Least Concern, OTS- Other Threatened Species, DDData Deficient

\section{Floral Composition of tropical forest over limestone}

A total of 41 floral species were recorded belonging to 18 plant families and 24 genera (Table 2). The most represented family was Moraceae ( $5 \mathrm{spp}$.), followed by Arecaceae (3 spp.), Sapotaceae (2 spp.), Euphorbiaceae (2 spp.), Araceae (2 spp.), Thymelaeaceae (2 spp.), and Apocynaceae (2 spp.). Family Lomariopsidaceae, Aspleniaceae, Gnetaceae, Annonaceae, Commelinceae, Fabaceae, Phyllanthaceae, Dipterocarpaceae, Malvaceae, and Cornaceae were each represented by a single species. Of the 41 floral species recorded, 6 are shrubs and 35 are trees. 
Table 2. List of plant species recorded from the forests over limestone of Guiuan Marine Reserve Protected Landscape and Seascape in Guiuan, Eastern Samar, Philippines.

\begin{tabular}{|c|c|c|c|c|c|c|c|}
\hline \multirow[b]{2}{*}{$\begin{array}{l}\text { Plant Group } \\
\text { Family }\end{array}$} & \multirow[b]{2}{*}{ Scientific Name } & \multirow[b]{2}{*}{ Common Name } & \multirow[b]{2}{*}{ Exsiccata } & \multirow[b]{2}{*}{ Habit } & \multicolumn{3}{|c|}{ Conservation Status } \\
\hline & & & & & $\begin{array}{l}\text { IUCN Red } \\
\text { List }\end{array}$ & $\begin{array}{c}\text { DAO } \\
2017- \\
11\end{array}$ & Endemicity \\
\hline \multicolumn{8}{|c|}{ Pteridophyta } \\
\hline Lomariopsidaceae & Cyclopeltis crenata (Fée) C. Chr. & Lukdo & $\begin{array}{l}\text { Obeña } 7111 \\
\text { (PBDH) }\end{array}$ & $S$ & - & - & $N$ \\
\hline Aspleniaceae & Asplenium vittaeforme Cav. & Lorog & $\begin{array}{l}\text { Obeña } 7091 \\
\text { (PBDH) }\end{array}$ & $S$ & - & NE & $N$ \\
\hline \multicolumn{8}{|c|}{ Spermatophyta: Gymnospermae } \\
\hline Gnetaceae & Gnetum gnemon L. & Bago & $\begin{array}{l}\text { Obeña } 7073 \\
\text { (PBDH) }\end{array}$ & $\mathrm{T}$ & LC & NE & $N$ \\
\hline \multicolumn{8}{|c|}{ Spermatophyta: Angiospermae } \\
\hline Annonaceae & $\begin{array}{l}\text { Monoon oblongifolium (C.B.Rob.) B.Xue \& } \\
\text { R.M.K.Saunders }\end{array}$ & Lapisan & $\begin{array}{l}\text { Obeña } 7164 \\
\text { (PBDH) }\end{array}$ & $\mathrm{T}$ & - & NE & $E$ \\
\hline \multirow[t]{2}{*}{ Araceae } & Aglaonema commutatum Schott & Pilako & $\begin{array}{l}\text { Obeña } 7089 \\
\text { (PBDH) }\end{array}$ & $S$ & - & - & $N$ \\
\hline & Rhaphidophora sp. & $\begin{array}{l}\text { No common } \\
\text { name }\end{array}$ & $\begin{array}{l}\text { Obeña } 7090 \\
\text { (PBDH) }\end{array}$ & $S$ & - & NE & - \\
\hline Pandanaceae & Unidentified & Bariw & & $S$ & - & - & - \\
\hline \multirow[t]{3}{*}{ Arecaceae } & Saribus rotundifolius (Lam.) Blume & Anahaw & $\begin{array}{l}\text { Obeña } 7040 \\
\text { (PBDH) }\end{array}$ & $\mathrm{T}$ & - & NE & $N$ \\
\hline & Heterospathe intermedia (Becc.) Fernando & Banga & $\begin{array}{l}\text { Obeña } 7076 \\
\text { (PBDH) }\end{array}$ & $\mathrm{T}$ & - & NE & $E$ \\
\hline & Caryota rumphiana Mart. & $\begin{array}{l}\text { Pugahan/ } \\
\text { Tagabunga }\end{array}$ & $\begin{array}{l}\text { Obeña } 7087 \\
\text { (PBDH) }\end{array}$ & $\mathrm{T}$ & LC & NE & $N$ \\
\hline Commelinaceae & $\begin{array}{l}\text { Tradescantia zebrina var. zebrina } \\
\text { Synonym: Zebrina pendula }\end{array}$ & $\begin{array}{l}\text { No common } \\
\text { name }\end{array}$ & $\begin{array}{l}\text { Obeña } 7092 \\
\text { (PBDH) }\end{array}$ & $S$ & - & NE & $N$ \\
\hline \multirow[t]{2}{*}{ Euphorbiaceae } & $\begin{array}{l}\text { Hancea wenzeliana (Slik) S.E.C.Sierra, Kulju \& } \\
\text { Welzen }\end{array}$ & Apanang & $\begin{array}{l}\text { Obeña } 7072 \\
\text { (PBDH) }\end{array}$ & $\mathrm{T}$ & - & NE & $E$ \\
\hline & Macaranga tanarius (L.) Müll.Arg. & Minunga & $\begin{array}{l}\text { Obeña } 7085 \\
\text { (PBDH) }\end{array}$ & $\mathrm{T}$ & - & NE & $N$ \\
\hline Phyllanthaceae & Bridelia glauca Blume & Anislag & $\begin{array}{l}\text { Obeña } 7102 \\
\text { (PBDH) }\end{array}$ & $\mathrm{T}$ & - & NE & $N$ \\
\hline Fabaceae & Wallaceodendron celebicum Koord. & $\begin{array}{l}\text { Banuyo/ } \\
\text { Salukigi }\end{array}$ & $\begin{array}{l}\text { Obeña } 7077 \\
\text { (PBDH) }\end{array}$ & $\mathrm{T}$ & - & VU & $N$ \\
\hline \multirow[t]{5}{*}{ Moraceae } & Artocarpus blancoi (Elmer) Merr. & Antipolo & - & $\mathrm{T}$ & - & $\mathrm{NE}$ & $E$ \\
\hline & Artocarpus rubrovenius Warb. & Tugop & $\begin{array}{l}\text { Obeña } 7147 \\
\text { (PBDH) }\end{array}$ & $\mathrm{T}$ & OTS & NE & $E$ \\
\hline & Ficus ampelas Burm.f. & Lanete & $\begin{array}{l}\text { Obeña } 7084 \\
\text { (PBDH) }\end{array}$ & $\mathrm{T}$ & LC & NE & $N$ \\
\hline & Ficus sp. (2) & Hagimit & $\begin{array}{l}\text { Obeña } 7082 \\
\text { (PBDH) }\end{array}$ & $\mathrm{T}$ & - & NE & - \\
\hline & Ficus sp. (1) & Dalakit & $\begin{array}{l}\text { Obeña } 7079 \\
\text { (PBDH) }\end{array}$ & $\mathrm{T}$ & - & NE & - \\
\hline \multirow[t]{2}{*}{ Thymelaeaceae } & Aquilaria cumingiana (Decne.) Ridl. & Agar/ Lapnisan & $\begin{array}{l}\text { Obeña } 7070 \\
\text { (PBDH) }\end{array}$ & $\mathrm{T}$ & VU & VU & $N$ \\
\hline & Gonystylus reticulatus (Elmer) Merr. & Batuan & - & $T$ & - & NE & - \\
\hline
\end{tabular}




\begin{tabular}{|c|c|c|c|c|c|c|c|}
\hline \multirow[b]{2}{*}{$\begin{array}{l}\text { Plant Group } \\
\text { Family }\end{array}$} & \multirow[b]{2}{*}{ Scientific Name } & \multirow[b]{2}{*}{ Common Name } & \multirow[b]{2}{*}{ Exsiccata } & \multirow[b]{2}{*}{ Habit } & \multicolumn{3}{|c|}{ Conservation Status } \\
\hline & & & & & $\begin{array}{l}\text { IUCN Red } \\
\text { List }\end{array}$ & $\begin{array}{c}\text { DAO } \\
2017- \\
11\end{array}$ & Endemicity \\
\hline Dipterocarpaceae & Shorea negrosensis Foxw. & $\begin{array}{l}\text { Lawaan na Pula } \\
\text { (Takuban) }\end{array}$ & $\begin{array}{l}\text { Obeña } 7122 \\
\text { (PBDH) }\end{array}$ & $\mathrm{T}$ & LC & VU & $E$ \\
\hline Malvaceae & Sterculia comosa Wall. & Balinad & - & $\mathrm{T}$ & - & $\mathrm{NE}$ & - \\
\hline Cornaceae & Mastixia sp. & Tul-anan & $\begin{array}{l}\text { Obeña } 7148 \\
\text { (PBDH) }\end{array}$ & $\mathrm{T}$ & - & $\mathrm{NE}$ & - \\
\hline \multirow[t]{2}{*}{ Sapotaceae } & Palaquium sp. (1) & Bagotambis & $\begin{array}{l}\text { Obeña } 7074 \\
\text { (PBDH) }\end{array}$ & $\mathrm{T}$ & - & $\mathrm{NE}$ & - \\
\hline & Palaquium sp. (2) & Nato & $\begin{array}{l}\text { Obeña } 7086 \\
\text { (PBDH) }\end{array}$ & $\mathrm{T}$ & - & NE & - \\
\hline \multirow[t]{2}{*}{ Apocynaceae } & Wrightia sp. & Hamor-awon & $\begin{array}{l}\text { Obeña } 7083 \\
\text { (PBDH) }\end{array}$ & $\mathrm{T}$ & - & NE & - \\
\hline & Alstonia scholaris (L.) R. Br. & Dita & $\begin{array}{l}\text { Obeña } 7081 \\
\text { (PBDH) }\end{array}$ & $\mathrm{T}$ & LC & $\mathrm{NE}$ & $\mathrm{N}$ \\
\hline \multicolumn{8}{|c|}{ Unidentified species } \\
\hline & Unidentified & Yakal & $\begin{array}{l}\text { Obeña } 7151 \\
\text { (PBDH) }\end{array}$ & $\mathrm{T}$ & - & - & - \\
\hline & Unidentified & Bago-eho & $\begin{array}{l}\text { Obeña } 7095 \\
\text { (PBDH) }\end{array}$ & $\mathrm{T}$ & - & - & - \\
\hline & Unidentified & Bagubahi & $\begin{array}{l}\text { Obeña } 7075 \\
\text { (PBDH) }\end{array}$ & $\mathrm{T}$ & - & - & - \\
\hline & Unidentified & Bayarong & $\begin{array}{l}\text { Obeña } 7078 \\
\text { (PBDH) }\end{array}$ & $\mathrm{T}$ & - & - & - \\
\hline & Unidentified & Dalunutan & $\begin{array}{l}\text { Obeña } 7080 \\
\text { (PBDH) }\end{array}$ & $\mathrm{T}$ & - & - & - \\
\hline & Unidentified & Amahoyan & - & $\mathrm{T}$ & - & - & - \\
\hline & Unidentified & Atipon & - & $\mathrm{T}$ & - & - & - \\
\hline & Unidentified & Bagnaw & - & $\mathrm{T}$ & - & - & - \\
\hline & Unidentified & Kuyakya & - & $\mathrm{T}$ & - & - & - \\
\hline & Unidentified & Lubi & - & $\mathrm{T}$ & - & - & - \\
\hline & Unidentified & Pamintaogon & $\begin{array}{l}\text { Obeña } 7128 \\
\text { (PBDH) }\end{array}$ & $T$ & - & - & - \\
\hline & Unidentified & Hambabalud & $\begin{array}{l}\text { Obeña } 7045 \\
\text { (PBDH) }\end{array}$ & $\mathrm{T}$ & - & - & - \\
\hline
\end{tabular}

*Vouchers, habit $(\mathrm{T}=$ tree, $\mathrm{S}=$ shrub, $\mathrm{H}=$ herb), Status based IUCN Red List of Threatened Species or DAO 2017-11 (NE = Not Evaluated, DD = Data deficient, OT = Other Threatened Species, $\mathrm{LC}=$ Least Concern, $\mathrm{VU}=$ Vulnerable, EN = Endangered, $\mathrm{CR}=$ Critically endangered), endemicity ( $\mathrm{E}=$ Philippine endemic, $\mathrm{N}=$ non-endemic). Sensu APG IV (2016). 


\section{Discussion}

\section{Terrestrial vertebrate diversity}

The present study is the only assessment of amphibians, reptiles, and mammals in Guiuan, and represent new island records for most of these taxa in Calicoan Island. The only other published assessment of fauna in GMRPLS is a survey of birds in Calicoan Island by Potter (1953) which recorded 51 species also listed in Table 1 with updated taxonomy. Among all the faunal species found and recorded, the following are notable for their uses, distribution, conservation and more.

\section{Notable animal species}

\section{Platymantis guentheri (Boulenger 1882)}

Gunther's wrinkled ground frog (Fig. 2A) is a widespread endemic species in the Mindanao PAIC known from Biliran, Bohol, Dinagat, Samar, Leyte and Mindanao (Diesmos et al., 2015). Nine adult individuals were hand-captured near Linao Cave in Brgy. Sulangan, a new record for Calicoan Island. The species is highly abundant and capable of thriving in second growth, degraded, and fragmented patches of habitat (Sanguila et al., 2016). Some of the species were caught from fallen logs but most of them were observed perching on karst substrates, then quickly jumping into the limestone crevices to escape once disturbed. The species is currently listed as Least Concern in the IUCN, but it is an important indicator of the health of the karst ecosystem since they are sensitive to environmental changes.

Cyrtodactylus sumuroi Welton et al. 2010

The first records of the Samar bent-toed gecko (Fig. 2B) were from the forests of Brgy. San Rafael, Taft, Samar Island (Welton et al., 2010), and this study presents a new island record for the species in Calicoan Island. One adult individual was hand-captured near Linao Cave in Brgy. Sulangan, while three individuals hiding in limestone crevices were captured in Brgy. Pagnamitan (Fig. 2C). It is not yet assessed in the IUCN Red List and DAO 2019-09 and may require further study to assess its conservation status.

\section{Varanus samarensis Koch et al. 2010}

The Samar water monitor was previously known only from Samar, Leyte and Bohol Islands (Welton et al. 2014), and this study presents a new island record for the species in Calicoan Island. An adult individual was seen in Brgy. Pagnamitan. Information about the ecology of this species is still lacking (Koch et al. 2010), and it is categorized as Other Threatened Species (OTS) under the DAO 2019-09.

\section{Hypsipetes philippinus saturatior (E. J. O. Hartert, 1916)}

The Philippine bulbul (Fig. 2D) is a Philippine endemic bird characterized by dominant olive brown plumage with a streaked rufous throat. Five subspecies are currently recognized in the Philippines (del Hoyo et al., 2020) categorized according to differences in color shade, boldness of shaft streaks on throat, and small differences in overall size 
(Kennedy et al., 2000). The subspecies H. philippinus saturatior can be found in east central and southern Philippines. An adult individual was netted in Brgy. Pagnamitan.

\section{Ptenochirus minor}

The lesser musky fruit bat (Fig. 2E) is dark in coloration with short, sturdy snouts that is endemic in the Mindanao PAIC. In Brgy. Pagnamitan, three male adult individuals and two male subadult individuals were netted. This species can easily be mistaken for Ptenochirus jagori but can be distinguished by their difference in forearm lengths. $P$. minor has a forearm length of 60-78 mm while P. jagori has 76-90 mm (Ingle and Heaney, 1992).

\section{Bullimus bagobus}

The Bagobo rat (Fig. 2F) is a large murid species widespread in the Mindanao PAIC. A male subadult individual was trapped in Brgy. Sulangan near Linao Cave. It can be differentiated from Rattus everetti by having a tail that is notably shorter than the combined length of the head and body, with a longer and moderately wide hind feet (Rickart et al., 2002).

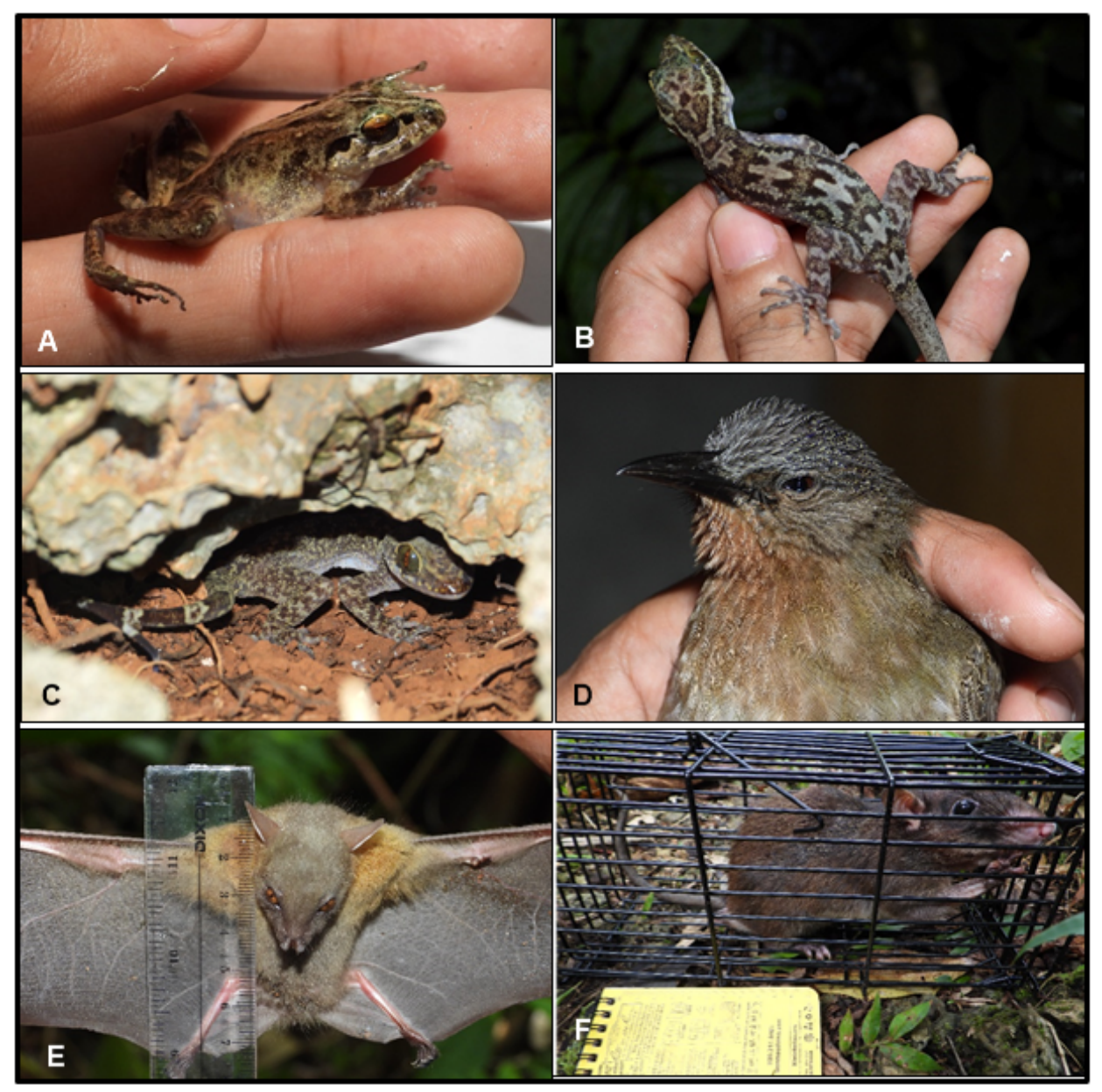

Fig 2. Some of the notable terrestrial vertebrate species from the forests over limestone of Guiuan Marine Reserve Protected Landscape and Seascape in Guiuan, Eastern Samar, Philippines: A) Platymantis guentheri, B \& C) Cyrtodactylus sumuroi, D) Hypsipetes philippinus saturatior, E) Ptenochirus minor, and F) Bullimus bagobus. 


\section{Plant diversity}

Species richness of tropical forests in Southeast Asia can range from 60 to 250 species per hectare (Losos and Leigh, 2004). The species richness of the tropical forest over limestone of Guiuan is below the aforementioned range. Generally, the forest is young, dominated by saplings and small diameter-sized trees. This could be attributed to the possibility that the forest over limestone in Guiuan is still recovering from the heavy rains and destructive winds of typhoon Haiyan which hit the island in 2013.

It was also observed that the area has low species richness as compared to other forest over limestones. Samar Island Natural Park (SINP) was recorded to have 212 timber species in the twenty-five 20x20 m plots (Quimio, 2016). In a similar study, 288 vascular plants were inventoried across the northeastern side of Mt. Tabunan, Cebu Island (Cadiz and Buot, 2010). In a study conducted by Lillo et al. (2019b), a total of 112 tree species were recorded from four 20x100-m permanent plots in Mount Lantoy key biodiversity areas in Argao, Cebu, Philippines. In Dinagat Island, a total of 432 native plant species were recorded across six different forest habitat types in which $58 \%$ were recorded from lowland evergreen forest, $16 \%$ from upper montane, $15 \%$ in forest over limestone, $6 \%$ in lower montane forest, $4 \%$ in mangrove forest, and $1 \%$ in beach forest (Lillo et al., 2019a). In a study conducted by Adam and Mamat (2005), they recorded a total of 1682 trees in a 0.75 ha plot. In the tropical forest over limestone in Xishuangbanna, China, a total of 100 tree species belonging to 74 genera and 31 families were recorded in the four 0.25 ha plots (Tang et al., 2011). Based from literature and comparisons, it would seem that floral species diversity in tropical forests over limestone varies greatly across different areas. The following is a short list of notable species of plant life found in the area.

\section{Notable plant life}

\section{Hancea wenzeliana (Slik) S.E.C. Sierra, Kulju \& Welzen}

This study reports a new locality record of this Philippine endemic tree species (Fig. 3). Based on literature, its native range is the island of Mindanao, Philippines. The occurrence of this tree species in Guiuan is not surprising since Samar is a constituent of greater Mindanao. Thus, this tree species seems to have a widespread distribution.

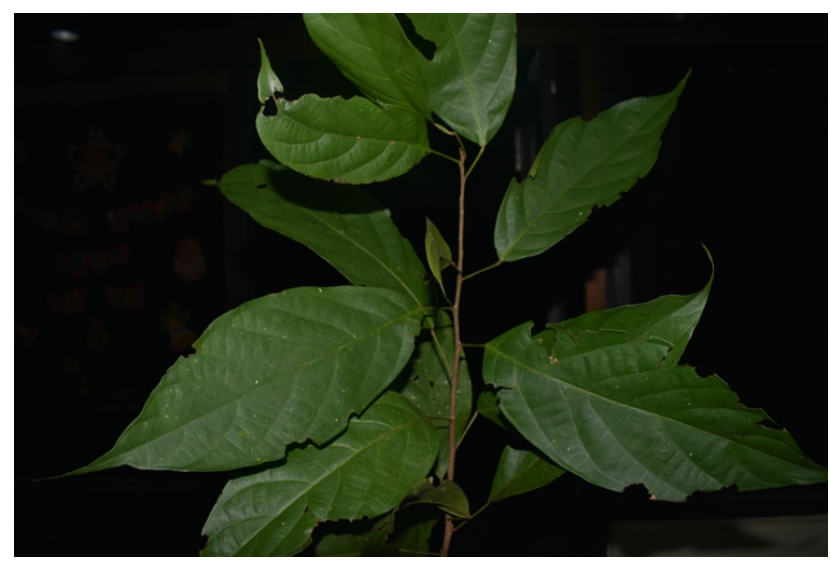

Fig 3. Vegetative structures of Hancea wenzeliana (Slik) S.E.C. Sierra, Kulju \& Welzen collected from the forests over limestone of Guiuan Marine Reserve Protected Landscape and Seascape in Guiuan, Eastern Samar, Philippines. 
Aquilaria cumingiana (Decne.) Ridl.

Commonly known as agarwood (Fig. 4), it is recorded as a well-known and important endemic tree species found in the Philippines and Indonesia. It is highly prized for its fragrant resin and utilized for various economic gains. Due to overharvesting leading to the rapid decline of its natural population, it is categorized as Vulnerable based on the DAO 2017-11 and the IUCN Red List. It is also protected under the Convention of International Trade in Endangered Species of Wild Flora and Fauna (CITES, 2004) regulation (Lee and Mohamed, 2016).

Wallaceodendron celebicum Koord.: Foxw. - in Philipp. J. Sc., Bot. 1907: 376; Koord., Suppl. Fl. Celebes. 1918: t. la, b.; Gilg - in E. \& P., Nat. Pflanzenfam. 1900: 30

This species is recorded to occur in two Asian-Tropical countries, which are the Philippines and Indonesia. It is identified as Vulnerable based on the DAO 2017-11.

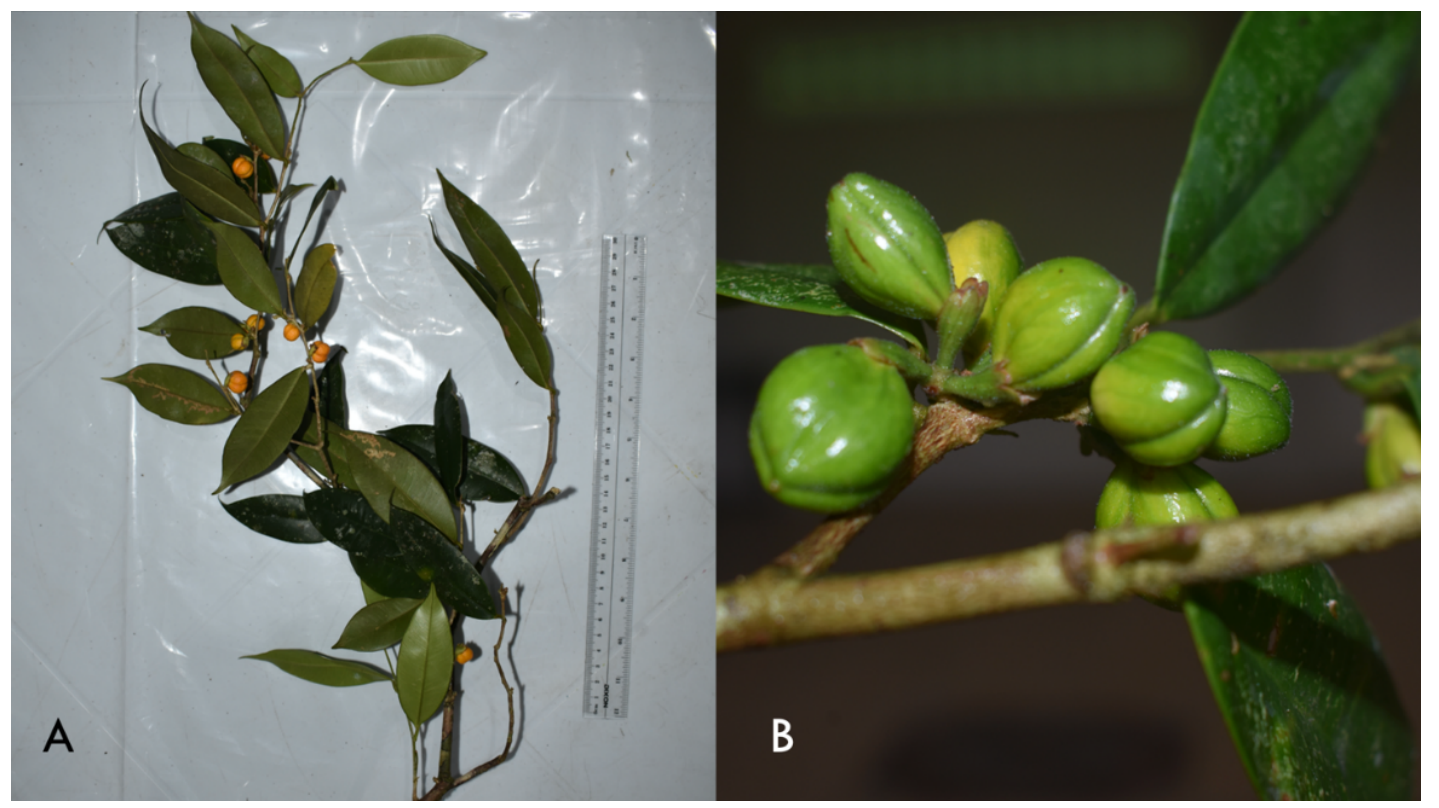

Fig 4. Vegetative and reproductive structures of Aquilaria cumingiana (Decne.) Ridl. collected from the forests over limestone of Guiuan Marine Reserve Protected Landscape and Seascape in Guiuan, Eastern Samar, Philippines. A) leaves and ripe fruits B) close up of the capsule.

\section{Conservation status of biodiversity in GMRPLS}

The present study shows that the forests over limestone in GMRPLS are home to numerous threatened species, highlighting the significance of the protected area for the conservation of these animals and plants. In both taxa, there are some discrepancies between their conservation statuses based on the IUCN Red List and the updated Philippine national Red Lists, DAO 2017-11 and 2019-09. In many cases, species listed as Least Concern in the IUCN Red List have no conservation status in the national Red List. This is because the lowest category in the Philippine Red List for fauna is OTS, which is more similar in description to the Near Threatened category of the IUCN Red List (Gonzalez et al, 2018). 
For the Samar water monitor, $V$. samarensis, it has not yet been assigned a conservation status by the IUCN perhaps because it was described only relatively recently by Koch et al. in 2010. However, this lizard is categorized in the Philippine Red List as OTS due to threats that include hunting for bushmeat and trade (BCSP, 2020). In some instances, the national conservation status may be higher than that of the IUCN status. Such is the case for the red lauan, Shorea negrosensis Foxw., and the reticulated python, Malayopython reticulatus Schneider 1801. For the former, more recent data used in the international assessment found that, though the population of this tree species may be in decline, it has a large enough extent of occurrence warranting a lower category (EDC, 2020). For the latter, it might be the opposite. The threat to this snake is lower internationally, but in the Philippines, it is highly persecuted or collected for trade (BCSP, 2020). This underscores the value of biodiversity monitoring to continuously update data that can serve as the basis for the formulation of environmental policies and protected area management strategies.

\section{Protected area management recommendations}

The native fauna of GMRPLS, particularly birds, have a great economic value in terms of ecotourism. Taylor et al. (2015) described the Eastern Visayas Region to be "deserving more attention from birdwatchers and ornithologists", therefore, stricter implementation of anti-poaching regulations should be upheld in GMRPLS. In a tourism development plan for Calicoan Island, Anore et al. (2019) proposed a community-based tourism approach. The region has been dubbed the surfing capital of Visayas and various surfing competitions plans may encourage more tourists which is related to the development of the area. It is recommended that sustainable ecotourism should be integrated into this approach, and the results of this present study could help inform local stakeholders on the status of the biodiversity and why conservation would be directly beneficial to ecotourism.

Integrating native and endemic tree species leads to species richness in natural tropical forests, and this should be incorporated into reforestation and plantation schemes for its conservation (Peque and Hölscher, 2014). It is recommended that propagation and reforestation efforts should be heightened especially but not limited to native and endemic tree species in Guiuan such as A. cuminigiana, W. celebicum, H. wenzeliana, S. negrosensis, Artocarpus blancoi (Elmer) Merr., Artocarpus rubrovenius Warb., and Monoon oblongifolium (C.B.Rob.) B.Xue \& R.M.K.Saunders. These efforts will benefit forests heavily affected by Typhoon Haiyan.

According to literature, other plant species recorded from the site have several uses, such as alternative food sources from the leaves of Gnetum gnemon L. Table 3 shows other uses of select plant species from GMRPLS. These plants may also be propagated in the area for the benefit of the local community. 
Table 3. Uses of select plant species recorded from the forests over limestone of Guiuan Marine Reserve Protected Landscape and Seascape in Guiuan, Eastern Samar, Philippines.

\begin{tabular}{|l|l|l|l|l|l|}
\hline Plant Group & \multicolumn{1}{|c|}{ Family } & $\begin{array}{c}\text { Scientific } \\
\text { Name }\end{array}$ & $\begin{array}{l}\text { Plant } \\
\text { Part }\end{array}$ & \multicolumn{1}{c|}{ Uses } & Reference \\
\hline Gymnosperm & Gentaceae & $\begin{array}{l}\text { Gentum } \\
\text { gnemon }\end{array}$ & Leaves & Blanched and braised in small bamboo pots & $\begin{array}{l}\text { Verheij and } \\
\text { Sukendar } \\
(1991)\end{array}$ \\
\hline Angiosperm & Araceae & $\begin{array}{l}\text { Aglaonema } \\
\text { commutatum }\end{array}$ & Leaves & Potential for use as natural antimicrobial agents & $\begin{array}{l}\text { Opryshko } \\
\text { et al. } \\
(2019)\end{array}$ \\
\cline { 2 - 6 } & Arecaceae & $\begin{array}{l}\text { Caryota } \\
\text { rumphiana }\end{array}$ & $\begin{array}{l}\text { Whole } \\
\text { plant }\end{array}$ & Ornamental & $\begin{array}{l}\text { Di } \\
\text { Benedetto } \\
(2006)\end{array}$ \\
\cline { 2 - 6 } & Commelinaceae & $\begin{array}{l}\text { Tradescantia } \\
\text { zebrina var. } \\
\text { zebrina }\end{array}$ & Leaves & $\begin{array}{l}\text { Treat skin conditions, common colds, and has inhibitory effects on cancerous } \\
\text { and non-cancerous cells }\end{array}$ & $\begin{array}{l}\text { Moehring } \\
(2013)\end{array}$ \\
\cline { 2 - 6 } & Thymelaeaceae & $\begin{array}{l}\text { Aquilaria } \\
\text { cuminigiana }\end{array}$ & $\begin{array}{l}\text { Leaves } \\
\text { and } \\
\text { bark }\end{array}$ & $\begin{array}{l}\text { Highly demanded in several countries, agarwood is further processed into } \\
\text { perfumes, incenses, and ornamental displays and used as a raw material in } \\
\text { traditional and modern medicines }\end{array}$ & $\begin{array}{l}\text { Lee and } \\
\text { Mohamed } \\
\text { (2016) }\end{array}$ \\
\hline
\end{tabular}

\section{Conclusion}

The present study provides vital information for future local and international conservation status assessments and plays a role in filling data gaps for many species living in such an understudied ecosystem such as forests over limestone. For example, some species recorded in this study represent new island or locality records. This includes $H$. wenzeliana and $C$. sumuroi, which are both not well-known and have not yet been categorized in local and international conservation status assessments. The new distribution records presented here contribute data that will be valuable in not only in conservation status assessments but also for management planning.

The Cave Management, Protection and Conservation Strategy and Action Plan (CMPCSAP) has laid out a framework for the conservation and sustainable management of Philippine caves, cave resources, and karsts, and it also has provisions for formulating and implementing policies regarding the sustainable use of cave and karst resources, as well as the sustainable management of selected caves and karsts as ecotourism areas (DENR-BMB, 2019). One of the study sites in the present study, Linao Cave in Brgy. Sulangan, Guiuan, is a popular local tourist attraction, the management of which could be improved based on these guidelines. Moreover, Anore et al. (2019) proposed a community-based approach in a tourism development plan for Calicoan Island due to its reputation as the surfing capital of the Visayas. The various surfing competitions held here annually may attract more tourists, which in turn could encourage more development on the island that should be closely monitored and strictly managed to conserve the biodiversity of the area.

\section{Acknowledgments}

The authors would like to thank DOST-PCAARRD and DOST-GIA for funding the program (no. N9A6323), and DOST-SEI for allowing ELC Villanueva to take part in this research program through the Career Incentive Program. The authors would also like to thank the GMRPLS-PAMB and DENR Region 8 for granting them the gratuitous permit (no. 2019-16) to conduct the study, the barangay councils of Baras, Ngolos, Pagnamitan, and Sulangan, and the kind people of Guiuan, 
especially Tito Rambakod, for their assistance and hospitality. We also thank the UPLB Institutional Animal Care and Use Committee for granting us permit no. CAS-2019-016.

\section{References}

Adam, J.H., Mamat, Z., 2005. Floristic composition and structural comparison of limestone forests at three different elevations in Bau, Kuching, Sarawak, Malaysia. J. Biol. Sci. 5(4): 478-485.

Alura, D.P., Alura, R.P.C., 2016. Regeneration of mangrove forest devastated by Typhoon Haiyan in Eastern Samar, Philippines. Int. J. Curr. Res. 8(6): 32373-32377.

Anore, N.J.P., Bersamin, C.J.E., Catapang, I.M.M., Gatinga, G.E.M., Gomez, M.A.M., Robles, S.J.L., Mercado, J.M.T., 2019. Tourism development plan for the Calicoan Island: a community-based tourism (CBT) approach. Journal of Tourism, Hospitality and Culinary Arts, 11(1), pp.65-81.

APG IV, 2016. An update of the Angiosperm Phylogeny Group classification for the orders and families of flowering plants: APG IV. Bot. J. Linn. Soc. 181(1): 1-20.

Arlott, N., 2018. Birds of the Philippines: and Sumatra, Java, Bali, Borneo, Sulawesi, the Lesser Sundas and the Moluccas (Collins Field Guides). Harper Collins UK.

BCSP. Biodiversity Conservation Society of the Philippines (Eds.), 2020. Philippine Red List of Threatened Wild Fauna Part I - Vertebrates. Biodiversity Management Bureau, Department of Environment and Natural Resources, Philippines.

BirdLife International, 2017. Cacatua haematuropygia. The IUCN Red List of Threatened Species 2017: e.T22684795A117578604. Retrieved on 23 December 2020 from https://doi.org/10.2305/IUCN.UK.2017-3.RLTS.T22684795A117578604.en.

Cadiz, G.O., Buot, I.E. Jr., 2010. An enumeration of the vascular plants of Mount Tabunan, Cebu Island, Philippines. Thailand Nat. Hist. Mus. J. 4(2): 71-77.

Canillas, R. 2018. eBird Checklist: https://ebird.org/checklist/S64212514. eBird: An online database of bird distribution and abundance [web application]. eBird, Ithaca, New York. Retrieved on December 23, 2020.

Canillas, R. 2018. eBird Checklist: https://ebird.org/checklist/S64212178. eBird: An online database of bird distribution and abundance [web application]. eBird, Ithaca, New York. Retrieved on December 23, 2020.

Canillas, R. 2019. eBird Checklist: https://ebird.org/checklist/S64202705. eBird: An online database of bird distribution and abundance [web application]. eBird, Ithaca, New York. Retrieved on December 23, 2020.

CITES. Convention on International Trade in Endangered Species of Wild Fauna and Flora. 2004. Consideration of proposals for amendment of Appendices I and II - Aquilaria spp. and Gyrinops spp. Proceedings of Thirteenth Meeting of the Conference of the Parties, Bangkok, Thailand.

Corales-Ultra, O.G., Peja, R.P. Jr., Casas, E.V. Jr., 2019. Baseline study on the levels of heavy metals in seawater and macroalgae near an abandoned mine in Manicani, Guiuan, Eastern Samar, Philippines. Mar. Pollut. Bull. 149:110549.

Cruz, R.V.O., Gonzalez, J.C.T., de Guia, A.P.O., Tiburan, C.L.J., Malabrigo, P.L.J., Balatibat, J.B., Cereno, R.P., Fernandez, D.A.P., Buhay, A.F.V., 2017. Vertebrate wildlife assessment, in: Manual on Biodiversity Assessment and Monitoring System for Terrestrial Ecosystems: How-To Guidelines. GIZ and DENR-BMB, Philippines.

Day, M.J., Urich, P.B., 2000. An assessment of protected karts landscapes in Southeast Asia. Cave Karst Sci. 27: 61-70.

Diesmos, A.C., Watters, J.L., Huron, N.A., Davis, D.R., Alcala, A.C., Crombie, R.I., Afuang, L.E., Gee-Das, G., Sison, R.V., Sanguila, M.B., Penrod, M.L., 2015. Amphibians of the Philippines, part I: checklist of the species. Proc. Calif. Acad. Sci. 62(20): 457-539.

Di Benedetto, A., Molinari, J., Boschi, C., Benedicto, D., Cerrotta, M., Cerrotta, G., 2006. Estimating crop productivity for five ornamental foliage plants. Int. J. Agric. Res. 1(6): 522-533. 
del Hoyo, J., Elliott, A., Sargatal, J., Christie, D.A., Kirwan, G. (eds.), 2020. Handbook of the Birds of the World Alive. Lynx Edicions, Barcelona, Spain. Retrieved on 30 March 2020 from http://www.hbw.com/.

DENR. Department of Environment and Natural Resources, 2017. Administrative Order 2017-11, Updated National List of Threatened Philippine Plants and Their Categories. Department of Environment and Natural Resources, Philippines. Retrieved on 07 October 2020 from http://www.bmb.gov.ph/index.php/e-library/laws-and-policies/denr-administrative-orders/dao-2017-2019?download=197:denr-administrativeorder-2017-11.

DENR. Department of Environment and Natural Resources, 2019. Administrative Order 2019-09, Updated National List of Threatened Philippine Fauna and Their Categories. Department of Environment and Natural Resources, Philippines. Retrieved on 07 October 2020 from http://www.bmb.gov.ph/index.php/e-library/laws-and-policies/denr-administrative-orders/dao-2017-2019?download=383:denr-administrativeorder-2019-09.

DENR-BMB. Department of Environment and Natural Resources - Biodiversity Management Bureau, 2019. Cave Management, Protection and Conservation Strategy and Action Plan (CMPCSAP) 2019-2028, Philippines. Retrieved on 07 October 2020 from https://www.bmb.gov.ph/index.php/caves/programs-caves.

EDC. Energy Development Corporation, 2020. Shorea negrosensis. The IUCN Red List of Threatened Species 2020: e.T33144A68074543. Retrieved on 07 October 2020 from https://dx.doi.org/10.2305/IUCN.UK.2020-1.RLTS.T33144A68074543.en.

Gonzalez, J.C.T., Layusa, C.A.A., Afuang, L.E., Duya, M.R.M., Heaney, L.R., Balete, D.S., Tabaranza, D.G.E., Española, C.P., van de Ven, W.A.C., Diesmos, A.C., Causaren, R.M., Diesmos, M.L.L., Lagat, R.T., Realubit, N.D.C., Sy, E.Y., Lit, I.L. Jr, Naredo, J.C.B., Lastica-Ternura, E.A., Pasicolan, S.A., Tagtag, A.M., De Leon, J.L., Lim, T.M.S., Ong, P.S., 2018. Review and update of the 2004 National List of Threatened Terrestrial Fauna of the Philippines. Sylvatrop 28: 73-144.

Hilvano, N., Nelson, G.L.M., Coladilla, J.O., Rebancos, C.M., 2016. Household disaster resiliency on Typhoon Haiyan (Yolanda): the case of Manicani Island, Guiuan, Eastern Samar, Philippines. Coast. Eng. J. 58(1): 1640007-1-25.

Ingle, N.R., Heaney, L.R., 1992. A key to the bats of the Philippine Islands. Fieldiana Zool. (NS) 69, 1-44.

Kennedy, R., Gonzales, P.C., Dickinson, E., Miranda Jr, H.C., Fisher, T.H., 2000. A Guide to the Birds of the Philippines. Oxford University Press.

Koch, A., Gaulke, M., Boehme, W., 2010. Unravelling the underestimated diversity of Philippine water monitor lizards (Squamata: Varanus salvator complex), with the description of two new species and a new subspecies. Zootaxa 2446(1), 1-54.

Lee, S.Y., Mohamed, R., 2016. The origin and domestication of Aquilaria, an important agarwood producing genus. Agarwood, 1-20.

Lillo, E.P., Fernando, E.S., Lillo, M.J.R., 2019. Plant diversity and structure of forest habitat types on Dinagat Island, Philippines. J. Asia Pac. Biodivers. 12(1): 83-105.

Lillo, E.P., Malaki, A.B., Alcazar, S.M.T., Nuevo, R.U., Rosales, R., 2019. Native trees on Mount Lantoy Key Biodiversity Areas (KBA), Argao, Cebu, Philippines. Philippine Journal of Science 148(2), 359-371.

Lin, I.I., Pun, I.F., Lien, C.C., 2014. "Category-6" supertyphoon Haiyan in global warming hiatus: contribution from subsurface ocean warming. Geophys. Res. Lett. 41 (23), 8547-8553.

Losos, E.C., Leigh, E.G. (Eds.), 2004. Tropical Forest Diversity and Dynamism: Findings From a Large-Scale Plot Network. Chicago University Press, Chicago. 688 pp. Maryna O., Halyna T., Lyudmyla B., Natalia K., Anna G., Vladimir T., Zbigniew O., 2019. Evaluation of the Antibacterial Activity of Ethanolic Extracts obtained from Aglaonema commutatum Schott and its cultivars against Citrobacter freundii. Agr.bio.div. Impr. Nut., Health Life Qual. 2019: 154-164.

Mendoza, A.B., Alura, D.P., 2001. Mangrove structure on the eastern coast of Samar Island, Philippines, in: Stott D.E., Mohtar R.H., Steinhardt G.C. (Eds.), Sustaining the Global Farm: Selected Papers from the 10th International Soil Conservation Organization Meeting, May 24-29 1999, West Lafayette. Purdue University and the USDA-ARS National Soil Erosion Research Laboratory, pp. 423-425.

Moehring, D.M., 2013. The anti-proliferative properties of Tradescantia zebrina. The FASEB Journal. 27(1). 
Myers, N., Mittermeier, R.A., Mittermeier, C.G., Da Foseca, G.A.B., Kent, J., 2000. Biodiversity hotspots for conservation priorities. Nature 403: 853-858.

Opryshko, M., Tkachenko, H., Buyun, L., Kurhaluk, N., Góralczyk, A., Tomin, V. and Osadowski, Z., 2019. Evaluation of the antibacterial activity of ethanolic extracts obtained from Aglaonema commutatum Schott and its cultivars against Citrobacter freundii. Agr.bio.div. Impr. Nut., Health Life Qual. (3): 154-164.

Peja, R.P., Alcober, J.P.G., Corales, O.G., 2018. Levels of nickel in Sargassum polycystum (Fucales, Sargassaceae) and Thalassia hemprichii (Alismatales, Hydrocharitaceae) in Manicani Island, Guiuan, Eastern Samar, Philippines. Phil. J. Nat. Sci. 21(2): 48-56.

Pelser, P.B., Barcelona, J.F., Nickrent, D.L. (Eds.)., 2011 onwards. Co’s Digital Flora of the Philippines. www.philippineplants.org.

Peque, D.P., Hölscher, D., 2014. Rare tree species in nurseries across the Visayas, Philippines. Int. J. Biodivers. Conserv. 6(7): 589-599.

Potter, N.S. III., 1953. The birds of Calicoan, Philippine islands. The Wilson Bulletin 65(4): 252-270.

Quimio, J.M., 2016. Floral composition and timber stock forest in the Samar Island Natural Park. Ann. Trop. Res. 38(2): 30-51.

Rickart, E.A., Heaney, L.R., Tabaranza Jr, B.R., 2002. Review of Bullimus (Muridae: Murinae) and description of a new species from Camiguin Island, Philippines. J. Mammal. 83(2): 421-436.

Sanguila, M.B., Cobb, K.A., Siler, C.D., Diesmos, A.C., Alcala, A.C., Brown, R.M., 2016. The amphibians and reptiles of Mindanao Island, southern Philippines, II: the herpetofauna of northeast Mindanao and adjacent islands. ZooKeys (624): 1.

Sodhi, N.S., Koh, L.P., Clements, R., Wanger, T.C., Hill, J.K., Hamer, K.C., Clough, Y., Tscharntke, T., Posa, M.R.C., Lee, T.M., 2010. Conserving Southeast Asian forest biodiversity in human-modified landscapes. Biol. Conserv. 143: 2375-2384.

Tang, J.W., Lu, X.T., Yin, J.X., Qi, J.F., 2011. Diversity, composition and physical structure of tropical forest over limestone in Xishuangbanna, Southwest China. J. Trop. For. Sci. 23(4): 425-433.

Takagi, H., Esteban, M., 2016. Statistics of tropical cyclone landfalls in the Philippines: unusual characteristics of 2013 Typhoon Haiyan. Nat. Hazards 80 (1): 211-222.

Taylor, J., Mate, E., Hutchinson, R.O., Eaton, J.A., 2015. Frontiers of the Philippines: Eastern Visayas. BirdingASIA, 24, pp.24-36.

Tuyet, D., 2001. Characteristics of karst ecosystems of Vietnam and their vulnerability to human impact. Acta Geol. Sin. 75: 325-329.

Verheij, E.W.M., Sukendar, 1991. Gnetum gnemon L. In: Verheij, E.W.M., and R.E. Coronel. 1991. Plant Resources of South East Asia 2. Edible Fruits and Nuts. PROSEA, Bogor, Indonesia.

Welton, L.J., Siler, C.D., Linkem, C.W., Diesmos, A.C., Brown, R.M., 2010. Philippine bent-toed geckos of the Cyrtodactylus agusanensis complex: multilocus phylogeny, morphological diversity, and descriptions of three new species. Herpetol. Monogr. 24: 55-85.

Welton, L.J., Travers, S.L., Siler, C.D., Brown, R.M., 2014. Integrative taxonomy and phylogeny-based species delimitation of Philippine water monitor lizards (Varanus salvator Complex) with descriptions of two new cryptic species. Zootaxa 3881(3): 201-227. 